\title{
MAPPING SHIP TRAFFIC IN NARROW CHANNELS WITH BENDS USING AUTOMATIC IDENTIFICATION SYSTEM (AIS) DATA
}

\author{
Jeffrey A. Oskamp, P.E., Moffatt \& Nichol, joskamp@moffattnichol.com \\ Eric D. Smith, P.E., Moffatt \& Nichol, esmith@moffattnichol.com
}

\section{INTRODUCTION}

Coastal, port, and waterway projects often require an understanding of the waterborne traffic in the site vicinity. Knowledge of what types of vessels transit near a project site, along with the vessel speeds and typical transit times/paths, can be valuable information to an engineer.

Often the best available information for vessel traffic can be obtained from Automatic Identification System (AIS) data. AIS data includes information about the vessel type, position, course, and speed (IMO, 2014).

Historic AIS data is available from a variety of free and commercial sources. Inside the United States, a large quantity of AIS data is available freely to the public from the United States' Coast Guard datasets.

AIS data can be summarized in a variety of tabular and graphic formats. For spatial planning and visualization, an intuitive format for communicating vessel traffic to nontechnical audiences is a vessel density map (e.g., Figure 1). Straightforward methods are available (BOEM/NOAA, 2015) for producing vessel density maps in relatively open water and away from sharp channel bends. This paper addresses challenges with preparing vessel maps in areas with narrow channels and around bends.

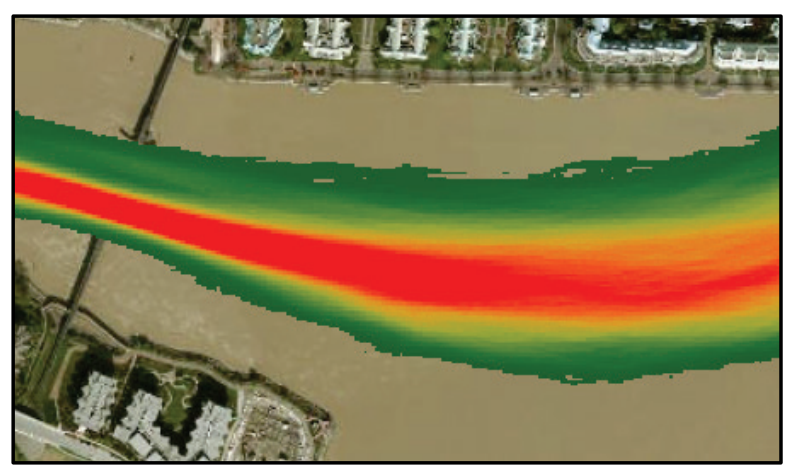

Figure 1 - Example Vessel Traffic Intensity Map (red=higher traffic; green=lower traffic)

\section{UNDERSTANDING THE CHALLENGE}

Taken as geospatial data, each AIS record is a point. Per IMO regulations, transiting vessels continually transmit a coded VHF radio signal. AIS datasets typically down sample this data to intervals varying from 1-5 minutes. A typical approach for generating vessel density maps involves connecting the time sequence "points" of a particular vessel for a particular voyage to make a polyline. Vessel density maps are created by aggregating a set of polylines in a way that identifies areas of higher and lower traffic. There are numerous ways of carrying out this analysis, including programs such as ArcGIS and MATLAB.

The limitation with this method is that it is based on turning points into polylines by connecting the points with straight lines. This straight-line assumption introduces considerable error to vessel density maps near tight channel bends, where lines connecting AIS data records on each side of the bend may bisect channel boundaries.

\section{ALTERNATE APPROACH}

This paper presents an approach for generating vessel density maps that better represents traffic around channel bends. The method avoids connecting individual AIS points with polylines:

- Each AIS data point is assigned a representative "swept path area", computed by multiplying the vessel's speed, beam, and AIS data time step. This represents the total area that the vessel would sweep through in space at its speed during the interval between AIS records.

- The AIS points are then binned into grid cells. The swept path areas for all AIS points in each grid cell are added together and divided by the surface area of the cell, which provides a value which can be understood as "number of vessels".

- If this approach is applied for a large dataset, the result is a grid, where each cell reports a number of vessels. Even though the cell values were not obtained by counting the actual vessels transiting each cell, on average (along the length of the channel), the grid reports the correct number of vessels in each cell.

- The vessel density map is then smoothed in the alongchannel direction to obtain the final density map.

This method produces a visual representation of vessel traffic that has reasonable quantitative accuracy for planning purposes. Figure 2 shows a sample of vessel intensities calculated using this method. The solution is based on synthetic data to allow for comparison with a hypothetical "exact" solution. The paper will include further validation of the method with synthetic data as well as illustration of the method with real world data.

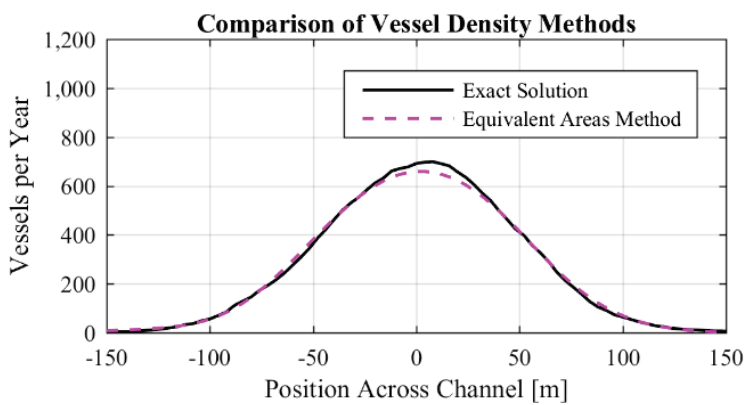

Figure 2 - Sample Results of Proposed Method

\section{REFERENCES}

Bureau of Ocean Energy Management (BOEM) and National Oceanic and Atmospheric Administration (NOAA) (2015): Tutorial: How to Build Vessel Density Maps with AIS.

IMO (2014): SOLAS, 6 ${ }^{\text {th }}$ Ed., Chapter V, Regulation 19, Paragraph 2.4. 